\title{
33 TOWARD UNDERSTANDING THE CAPABILITY CYCLE OF SOFTWARE PROCESS IMPROVEMENT: A Case Study of a Software Service Company
}

\author{
Yu Tong \\ Lingling Xu \\ Shanling Pan \\ Department of Information Systems \\ National University of Singapore \\ Singapore
}

\section{ABSTRACT}

The emergence of a service economy facilitates proliferation of software service companies (SSCs), small firms offering various software services such as software maintenance, testing, and customization. Given the unique characteristics of SSCs (i.e., smallscale projects, limited resources, strong customer dependency, etc.), software process improvement (SPI) in SSCs faces additional challenges. Based on Helfat and Peteraf's framework of a dynamic capability lifecycle, this study aims to understand how dynamic capabilities are developed to facilitate SPI implementation, and how these capabilities can be maintained and transformed in a changing context. An in-depth interpretive case study was conducted in a SSC, namely SGSC, which is the an offshore software service center of a leading U.S. document management company, Xerox Corporation. Drawing insights from the framework, this study demonstrates the evolution of key resources and dynamic capabilities as well as their impacts on the success of the SPI project.

Responding to the ownership transfer from Xerox to Fuji Xerox group in 2005, the center decided to implement new SPI process. As shown in Figure 1, three capabilities

Tong, Y., Xu, L., and Pan, S., 2008, in IFIP International Federation for Information Processing, Volume 267, Information Technology in the Service Economy: Challenges and Possibilities for the $21^{\text {st }}$ Century, eds. Barrett, M., Davidson, E., Middleton, C., and DeGross, J. (Boston: Springer), pp. 379-381. 


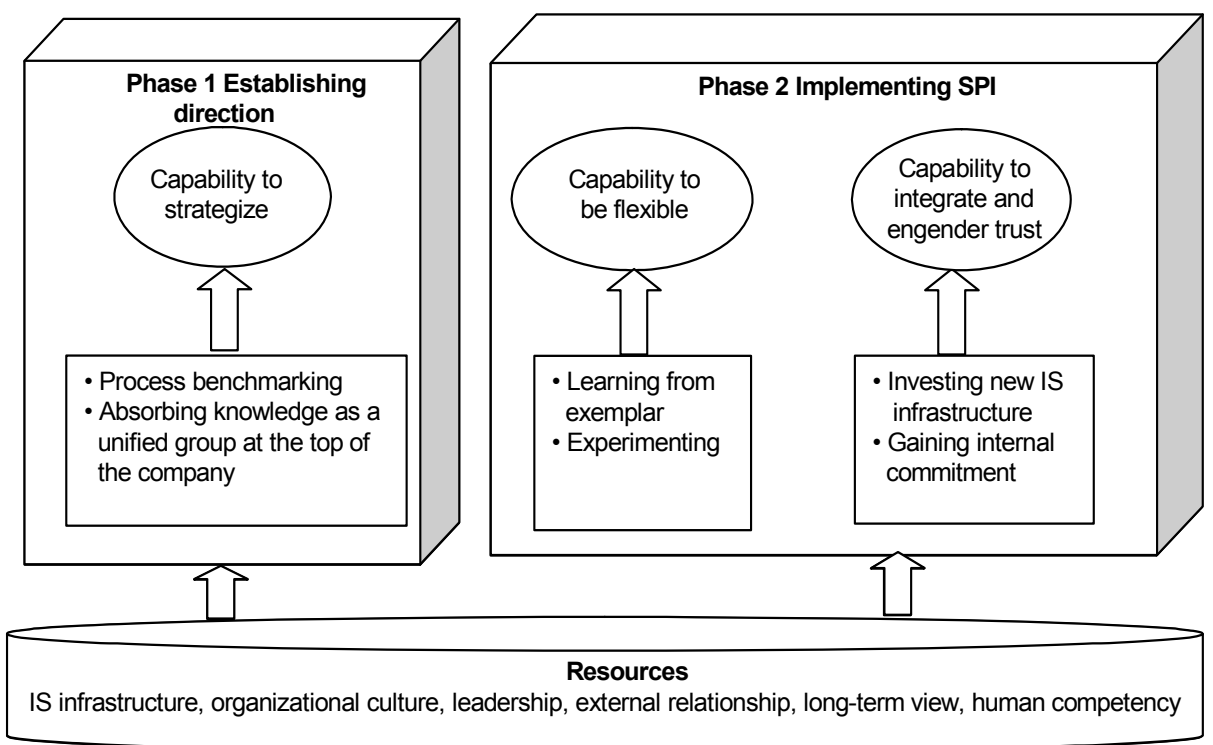

\section{Figure 1. Capability Development}

(i.e., capability to strategize, capability to be flexible, and capability to integrate and engender trust) were built up during the first two phases (i.e., establishing direction and implementing SPI). Development of each capability was accomplished through some actions with the support of various resources.

After development, capabilities can evolve over time (Zollo and Winter 2002). Two capabilities (capability to strategize and capability of integrate and engender trust) were maintained through regular exercise, which helps to refresh the company's memory and facilitate the creation of embedded knowledge. Besides being maintained, capabilities may also branch into different forms when external factors have a strong impact to alter the current development trajectory (Helfat and Peteraf 2003). At SGSC, ownership transfer resulted in a dramatic change in terms of external relationships and internal structure. With changes in the supporting resources, capabilities built up in previous phases were further transformed into different branches. Capability to be flexible was renewed because of better communication and sharing resources with external partners. Capability to strategize was also renewed after obtaining a long-term view from the new parent company. Figure 2 summarizes the life cycle of three capabilities in this study.

By integrating theoretical perspective with empirical evidence, this paper contributes to both researchers and practitioners. For researchers, it constitutes one of the first empirical studies to extend Helfat and Peteraf's general framework of the capability life cycle by demonstrating how capabilities can be transformed through altered resources over time. Moreover, this study advances the SPI literature by suggesting a conceptual framework for SPI implementation in a small-scale, service-oriented company. Practically, this study provides invaluable suggestions to managers on how firms can successfully implement SPI strategy when facing dramatic changes in the external environment or internal structure. 


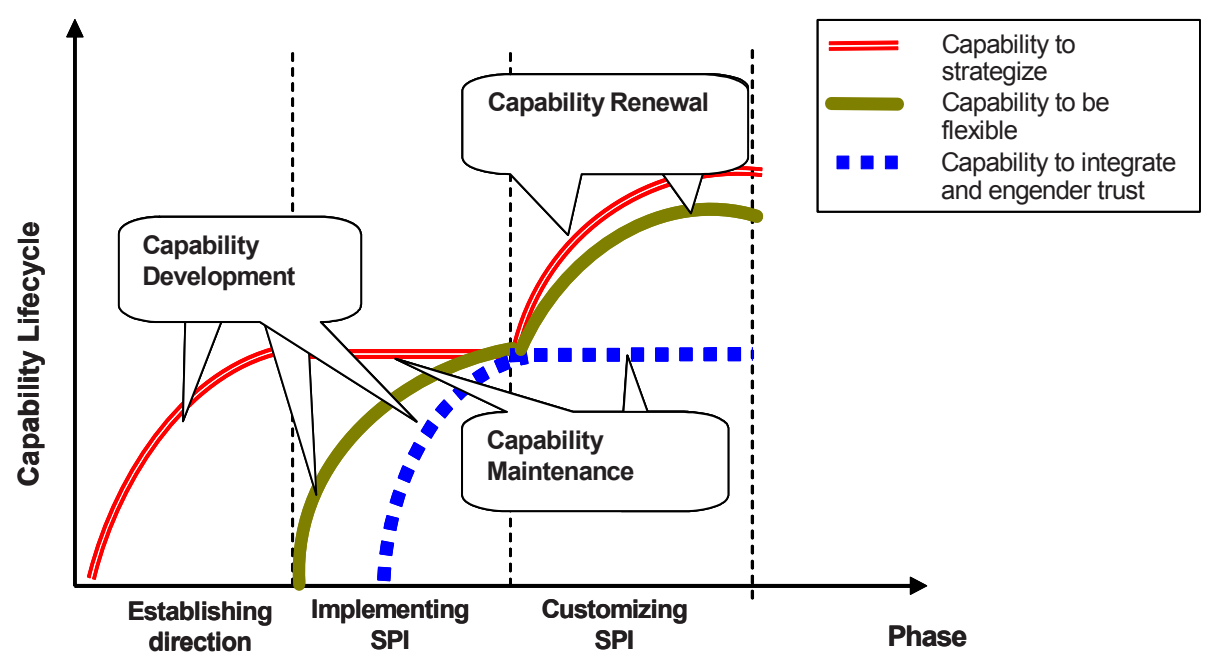

Figure 2. Capability Life cycle

\section{References}

Helfat, C. E., and Peteraf, M. A. 2004. “The Dynamic Resource-Based View: Capability Lifecycles," Strategic Management Journal (24:10), October, pp. 997-1010.

Zollo, M., and Winter, S. G. 2002. "Deliberate Learning and the Evolution of Dynamic Capabilities," Organization Science (13:3), pp. 339-351.

\section{About the Authors}

Yu Tong is a Ph.D. candidate in the Department of Information Systems at the National University of Singapore. Her research interests include IT adoption and assimilation in organizations, virtual team management, and IT-mediated marketing communications. She can be reached by e-mail at tongyu@comp.nus.edu.sg.

Lingling $\mathbf{X u}$ is a Ph.D. candidate in the Department of Information Systems at the National University of Singapore. Her research interests include human-computer interaction, social commerce, and IT adoption and implementation. She can be reached by e-mail at xulingling@, comp.nus.edu.sg.

Shanling Pan is the coordinator of the Knowledge Management Laboratory in the Department of Information Systems, School of Computing, National University of Singapore. Dr. Pan's primary research focuses on the recursive interaction of organizations and information technology (enterprise systems), with particular emphasis on issues related to work practices, culture, and structures from a knowledge perspective. 